\title{
An exploratory study of Mental Health and wellbeing in the Spiti Valley
}

\author{
Shraddha Vora \\ Research Scholar in Psychology, JJT University, Rajasthan. \\ Corresponding author: Shraddha Vora \\ Email - shraddhavora8@gmail.com
}

The Spiti valley is a remote, high altitude desert in the district of Lahaul and Spiti, Himachal Pradesh with approximately 11,852 inhabitants across 62 distinct villages. Tibetan Buddhism is the dominant religion, with pre-Buddhist Bon traditions remaining strongly ingrained in the culture. It is also known for its exotic wild life and rich flora and fauna. It is a designated tribal area and as such receives extra support from the Central Government of India [1-3]. The cultural landscape consists largely of Buddhist monasteries and mud houses, some of which as old as 10th century AD largely comprise the cultural landscape of the valley. It is only since three decades that the valley is open to tourists. In addition, Spiti valley is cut off from the rest of the world for 6-8 months of the year due to extreme weather conditions. The temperature in winter drops to $-20^{\prime} \mathrm{C}$ to $-30^{\prime} \mathrm{C}$. All these conditions make the life of people in Spiti one of the harshest living conditions in the world [4]. The study is an attempt to explore mental health in the valley with the rapid development and exposure of the valley inhabitants to the outer world.

\section{Methods}

The researcher spent some time understanding the local culture, life and religion of the locals. Based on interactions with the local NGO Ecosphere, a semi structured interview was designed. The interviewees were chosen to represent a number of viewpoints of well-informed groups: government employees, allopathic physicians, Amchis, nurses, teachers, an expatriate Special Educator, local members of the tourist industry, local community leaders, and senior nuns. Selection was also sensitive to age, gender and location in the valley. 9 interviews were conducted in Hindi and then translated and transcribed in English; 10 were conducted in English. Interviewees were assured that the purpose of the study was not to morbidify the current situation and that the happiness and mental wellbeing of the community was highly acknowledged.

\section{Themes of the interview}

\section{General health and wellbeing}

There exists a network of Amchis, practitioners of traditional local medicine (Soharigpa), alongside two Community Health Centres staffed by allopathic physicians in Kaza, the administrative capital, and Tabo. Interviewees reported that the community of people living in the Spiti valley is particularly healthy and cohesive. Despite this there is a minority of people who have developed what we would recognise to be mental health problems. The mental health situation is a consequence of the interaction between environmental, cultural, linguistic and spiritual elements (an example of the Buddhist philosophical concept of Multiple Causality). These elements have both negative and positive influences on mental health in society [5].

\section{Cultural Context}

Most interviewees mentioned being unaware of the meaning of the term 'mental health'. Some interviewees synonymously used the word 'depression' to refer to any mental health problem, whereas some just mentioned that mental health problems do not fit in the valley culture. Both interviews and our perceptions indicate that people are generally happy here. There is a very strong sense of community and the social fabric 
within and between families is very tight. The primary way of coping with any challenges is through this close network. In this way, traditional society and culture seems to preserve and foster wellbeing and good mental health. However, another integral aspect of the culture is that people are not very emotionally expressive and consequently often do not share their worries or concerns. This reserved and introverted culture can have a negative impact upon mental health of individuals; this concern was explicitly expressed. There also appears to be a linguistic (in addition to cultural) aspect to not expressing emotional concerns: the terms mental health, depression and even Luung (see below) are not a part of the lexicon. Finally, a particularly interesting finding was that almost all interviewees stressed there is little or no social stigma associated with mental health or Luung as compared to Indian/western society. One interviewee reported that such a diagnosis, far from generating stigma and ostracism, was met with sympathy and sensitivity from the community.

\section{Traditional Approaches to Mental Health}

The primary form of seeking help for medical and health concerns is by reference to the Amchi or the Gur. The Gur is a person who channels a La (Hindi: Devta), the local God, to determine the nature of the illness, treat the problem themselves, or direct the patient for further treatment. Occasionally the outcome of the ritual is advice to seek medical help from the Community Health Centre. Most often they are referred to a local practitioner of traditional medicine, an Amchi. The Amchi views illness in context as a result of many causal factors, one being the negative karma of the individual. The level of training and expertise varies between Amchis; some are trained in Tibetan Medical School whereas for some it is practised by family lineage. Amchis understand mental health problems to be part of 'Luung'. Luung is the condition of an imbalance in the wind element in the body. Amchis we interviewed mentioned 45 different types of Luung problem. Diagnosis is through history-taking, study of behaviour, examination of nerves, tongue and by the taking of the pulses. The grade of treatment depends on severity and type of symptoms. The primary treatment options rely on traditional herbal medicines derived from different plants across the valley5. Procedural options are sometimes used dependent on the individual, often if their condition is more severe, or when the necessary plants are unavailable. Two of the Amchis mentioned using what we recognised to be talking based therapies to treat the patient and support the family. They often explained and helped the patient to identify, process and deal with negative thoughts and emotions. This is an interesting example of fusing traditional and more modern methods to great effect. It is not clear how widespread this practice is across all the Amchis. Thus, the relationship between Luung, mental health and spiritual belief is complex and warrants further research.

\section{Allopathic Approaches to Mental Health}

Engagement in allopathic medicine is often the last form of treatment that people will seek for any health problem (although this may be changing). As a result, people will present with fairly developed and severe conditions. According to the Community Health Centre, people will not seek help for emotional or mental health issues due to lack of vocabulary and an idea that they don't view it as a problem for which they need to seek help. Thus, patients with mental health problems will largely present when they have manifested psychosomatic symptoms: insomnia, refusal to eat or drink, severe non-specific pains. Treatment will be focused on rectifying the physical symptoms. No mention of talk based therapies was made during interviews with allopathic practitioners and there are no psychiatrists, psychologists or counselling services in the valley.

\section{Gender and Mental Health}

In terms of gender, women play a large role in the society. They bear greater responsibility than men within the family and it is generally women who have the greater workload. They do almost all the agricultural work and play a central role in cultural festivities. Women are seen as strong but interviewees mentioned that emotional problems are more prevalent in the female population. Amchis report Luung patients to be disproportionately female. They often seek medical help for psychosomatic symptoms, which some attributed to their over-thinking and more sensitive nature. It is also possible that these are manifestations of 
the greater burden women shoulder. Though this is the general trend, it was brought to our attention that women's roles vary village by village.

\section{Age and Mental Health}

When questioned about age-related trends, both Soharigpa and allopathic practitioners cited the 40-50 year old or middle aged group as the greatest sufferers of mental health and Luung problems. This was explained using ideas of high workload, anxiety about children's educations and futures, anxiety about their own health in the future, and finally the high social responsibility of this age group. These pressures are therefore resulting in mental health issues, mental health-related Luung and also hypertension-related Luung. High rates of Luung problems in the older population were also mentioned. An Amchi drew attention to the emotional and psychological effects of chronic illness in older people: depression-type symptoms and anxiety about death. Suicide is most commonly associated with young people.

\section{Children and Mental Health}

We found that diagnosed mental health and Luung problems in children and young people are very rare. However we have been made aware of psychological concerns particularly associated with the education system. Traditionally, the second son in a family and some girls would be admitted to religious institutions (Buddhist nunneries and monasteries) from a young age. The existing institutions are still admitting children but the recent trend is away from this and towards full time education. One concern raised by both a local teacher and the Special Educator is that children as young as 4 years are leaving their families, whether to join religious institutions or boarding schools. They suspect this is having a profound and long lasting psychological impact on emotional development and wellbeing. Separation anxiety and effect on the family was also mentioned. The Special Educator also drew attention to the fact that the early years curriculum does not include any learning about their surroundings and Spitian culture, history and geography. She is concerned that this does not allow them to develop a healthy sense of self and belonging, potentially leading to high risk of mental health problems. In addition, academic pressure is resulting in a dread of going to school and high levels of anxiety. Fears about Karmic outcomes and other religious notions has resulted in children sharing with the special educator about bad dreams they have had. Another interviewee, an Amchi also reported concerns about increasing use of computer games and mobile devices instead of physical activity and sports. Finally, concern was expressed about minimal, poor parent-child interaction: parents rarely take an interest in what their child has learnt at school or what they have done during the day. It was therefore our impression that though wellbeing is generally good in the valley, there are some concerns relating to children's development.

\section{Alcohol and Mental Health}

Alcohol plays a significant and legitimate role in the traditional culture of the valley: within festivities; throughout the harsh winter; socially alongside the playing of cards amongst men. People use it to become more socially open and emotionally expressive - indeed it is used in this way across the world. Regular, open and moderate consumption is therefore an accepted norm; alcohol has traditionally been brewed at home in the form of Arak and Chang, which are both produced from the local staple, barley. Due to this accessibility, it is often used as first line analgesia to self-manage minor to moderate pain. It is within this context that a rising concern about alcohol use has been voiced in almost all our interactions. One idea has been that the prevalence within normal culture has allowed, in some cases, a gradual progression to more dependent use. Others have explained the rise in dependent usage by its use as an emotional coping mechanism in the absence of a culture of sharing such issues, either with close relatives or professionals. With the increases in stress and pressures due to the changing society discussed later, this is an increasing trend. Dependent alcohol use is almost exclusively an issue amongst adult males. Dependence, as indicated by anecdotal reports is estimated to be as high as $20 \%$ in some villages [6]. The Community Medical Centre, Kaza, sees around 3 patients per month with serious chronic conditions as a direct result of long term heavy alcohol use: liver cirrhosis; oral, stomach and liver cancers; serious withdrawal symptoms. The village of Rangrik is pejoratively nicknamed the Village of Widows due to the estimated 60 women widowed by alcoholism, an unprecedented rate for a settlement of population of 5007 people. One interviewee suggested that alcohol- 
related physical and emotional abuse toward spouses has occurred but on a very rare basis. Legal divorce is not a practice here but disruption of family and marriage due to alcohol is occurring, again on a rare and undocumented basis. There has been no concern expressed about alcohol related abuse towards children or any profound effect of alcohol in the family on children. In Kaza, a local women's group has already begun to work to reduce dysfunctional alcohol use and successfully lobbied the administration to ban public gambling. This is definitely an area ripe for research, whether clinical, anthropological or sociological, and whilst we have made attempts to accurately capture the trend, this is an incomplete picture of a complex phenomenon.

\section{Suicide}

Suicide is an understandably sensitive subject in the valley. Interviewees reported being concerned about the recent trend of isolated incidents 4 suicides in the last 12 months were reported. It is worth noting that a number of interviewees mentioned that the current rate is low and that a spike in the number of suicides actually occurred during the years 2005-2008. During this period in a single village, 4 suicides were reported of which 3 were in the 20-30 age group. We did not find reasons specific to this time frame as explanation. Those not familiar with the allopathic medical concepts of depression and mental health doubted the connection between Luung problems and suicide. This is perhaps because people view time of death within a spiritual and astrological context: that death is pre-determined and therefore independent of Luung. This fatalistic acceptance may help the family cope afterwards.

\section{Seasonal Fluctuation and Mental Health}

There is huge seasonal variation in lifestyle in Spiti owing to the climatic extremes. With phenomena such as Seasonal Affective Disorder (SAD) and seasonal fluctuations in anxiety and depression seen in other countries, we wanted to investigate any link between season and mental health here. Overall, these phenomena were not reported at all. Summer is the time of hard work here: society is primarily geared towards cultivation of the subsistence crop of barley, and the cash crop of green peas. This also applies to the tourist industry, particularly in Kaza, as tourists visit almost exclusively for 3 months of the year. During the height of summer, it was observed that women work in the fields for up to 12 hours per day. Winter is the time of relaxation. Despite very harsh weather conditions - temperatures below $-30^{\circ} \mathrm{C}$ and $3-8$ feet of snow isolating many of the villages for 3 months - society is very well adapted. People remain inside around their tandoors drinking tea, praying and talking. Children still attend school but the adults welcome this as a time of relaxation and far lower stress levels than the sometimes frenzied summer. It is also interesting that this is a time of great social and cultural activity as almost every day a different family in the village holds a social function. All marriages and many festivities are celebrated during winter. Thus, in relation to mental health it would be fair to say people are happier and live with far less stress and anxiety in the winter. When asked in both interviews and informal conversations with local people "Do you prefer winter or summer?" all answer winter. Therefore, even though a SAD-type phenomenon does not exist here, the picture is complex and varies across villages [7].

\section{Changing Society and the Future}

Spitian society is changing rapidly, especially in Kaza. Traditionally, the pace of life has been slow with "very little hustle and bustle" and a lifestyle geared around subsistence farming and weaving. This relaxed approach is possibly due to the culture of acceptance stemming from Buddhist philosophy. Stress levels have been low. The introduction of the market economy, technology and tourism is changing this. A shift from a traditional community-orientated society towards a more western individualistic culture is slowly occurring. Interviewees believed these attitudinal changes will increase competition and therefore pressure in many aspects of people's lives, particularly in education and income. Spitian society has always been economically fairly egalitarian; we assume this has been beneficial for mental health [8] due to lower levels of financial stress. The influx of tourists to the valley has very recently led to a development of illegal drug use, a phenomenon which has previously been non-existent. One interviewee estimated drug use amongst young people at $10 \%$. More Spitians are now leaving the valley, first for higher education, and then for business and better job prospects. Although currently a small scale social trend, it is predicted this will increase over 
the next decade and this will profoundly change the very tight social fabric. Indeed, already concerns are being voiced about loneliness in old age, which would have been unheard of within the traditional strong Spitian family structure. These trends towards more mental health issues have been recognised by the local Amchis, who say they are concerned Luung problems are increasing. It is however worth noting that one local Amchi drew attention to the fact that Luung problems have always existed, as documented by Tibetan medicine books, so mental health problems are not being primarily driven by these societal changes.

\section{Conclusions}

The Additional District Commissioner [9] told us that the gem of Spitian society was its happiness and sense of community and that this is both cultural and due to the Buddhist principles of compassion and peace. There remain linguistic and cultural barriers to the recognition, expression and effective discussion of mental health concerns. However, promising progress is being made around addressing alcohol use, the needs of elderly people and also the incorporation of some talking based therapies into the Amchi practices. In this way, a western-trained Spitian nurse explained that she thought the balance between science and spirituality is very good. People use spiritual, traditional Soharigpa and allopathic methods in conjunction to resolve their health concerns, usually very effectively. The strong social fabric is beneficial both preventatively for mental health of the population and in supporting those who fall sick. Thus the mental health trends and how Spiti deals with these challenges is an area very ripe for further research, particularly of a more quantitative and systematic nature.

\section{Authors' Acknowledgements}

The author is grateful to Ishita Khanna and team Ecosphere, a local NGO in Spiti Valley, Michael Heckenberg and Shannon Bernard Healey for their unconditional support and inputs through this project. We also thank all the interviewees for their valuable contributions.

\section{RECOMMENDED REFERENCES}

1. Estimated population by local official as part of 2010 Census of India

2. Bajpai SC. Lahaul - Spiti - A forbidden land in the Himalyas - Indus Publishing company, New Delhi, 1987.

3. Sikka S, Chaudhary C. Climate change in western himalaya impacts, mitigation and adaptation of historic earthen structures. COMOS Scientific Symposium - Malta; Changing World, Changing Views of Heritage ; 2009.

4. Gill MS. Himalayan Wonderland: Travel in Lahant Spiti, Delhi ; 1972.

5. Wind in Spitian/Bhoti is 'lu'; it is thought that Luung is a derivation of this

6. Devi et al (2013) Study on Ethnomedicinal Plants of Kibber Wildlife Sanctuary: a Cold Desert in Trans Himalaya, India; Journal of Medicinal Plants Research; 7(47): 3400-3419

7. Reports of at least 10 male individuals from a total population of 114 in Hikkim. This is not verified nor is necessarily a representative rate for other villages or the valley overall

8. Anecdotal. Reportedly 80 women widowed by alcohol in the upper valley, around 60 of which are in Rangrik. This population figure is estimated by locals and does not include the boarding students at Munseling School

9. The head of the Civil Authority in Spiti. 